\title{
Prevalence of Pediatric Onset Multiple Sclerosis in Saudi Arabia
}

\author{
Reem Bunyan, ${ }^{1}$ Ghada Al Towaijri, ${ }^{2}$ Hessa Al Otaibi, ${ }^{3}$ Abid Kareem, ${ }^{4}$ Hussein Algahtani, ${ }^{5}$ \\ Mousa Al Mejally, ${ }^{6}$ Ali Almubarak, ${ }^{7}$ Saad Alrajeh, ${ }^{8}$ Edward Cupler, ${ }^{9}$ Shireen Qureshi, ${ }^{10}$ \\ Sadaga. Alawi, ${ }^{11}$ Mamdouh Kalakatawi, ${ }^{12}$ Yaser Al Malik, ${ }^{13,14,15}$ Shahpar Nahrir, ${ }^{16}$ \\ Adel Alhazzani, ${ }^{17}$ Ashraf El-Metwally, ${ }^{18}$ Sahar Shami, ${ }^{19}$ Samah Ishak, ${ }^{19}$ \\ Hajer Almudaiheem, ${ }^{20}$ Ahmed Al-Jedai, ${ }^{20}$ and Mohammed AlJumah ${ }^{2}$
}

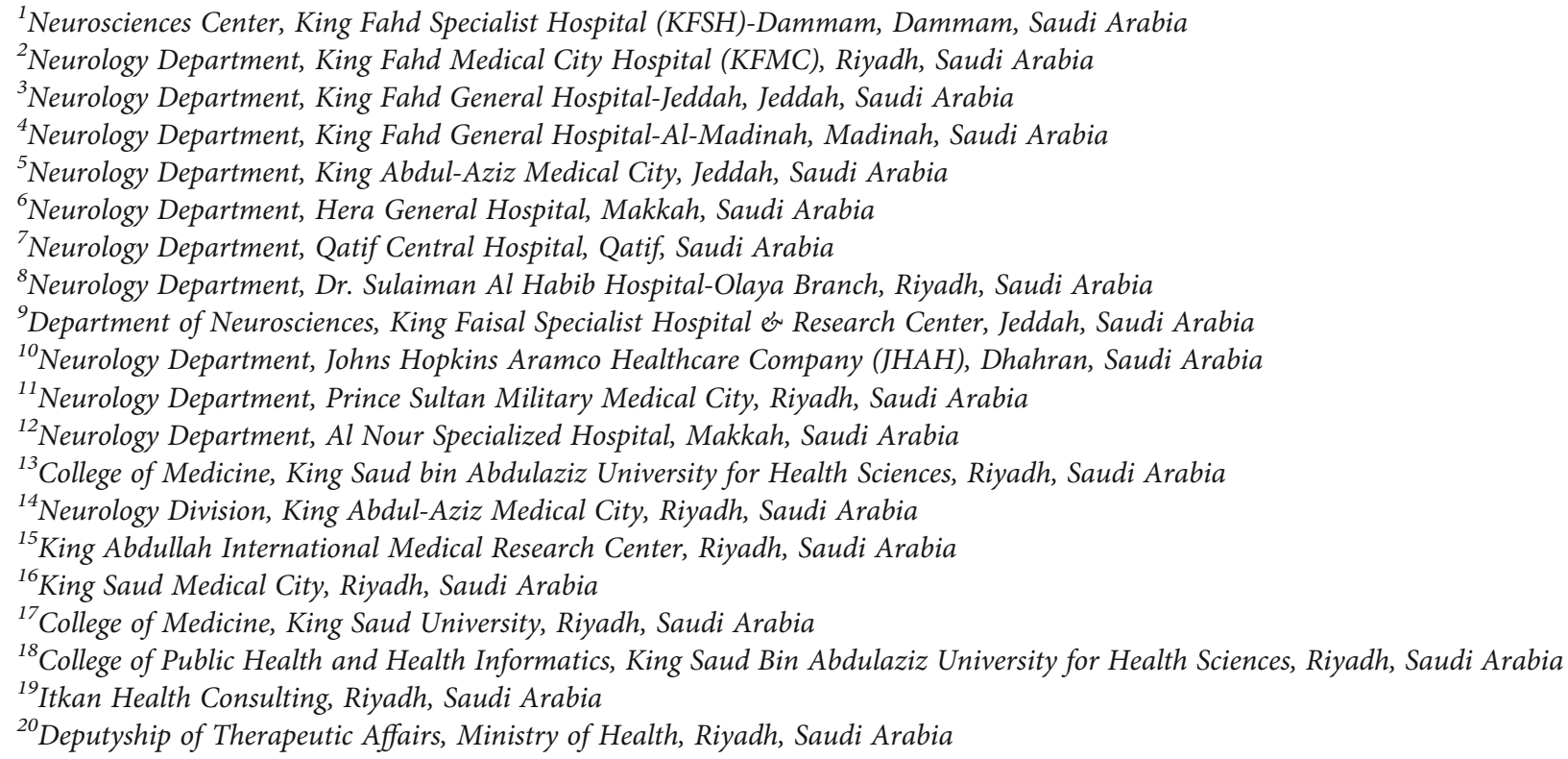

Correspondence should be addressed to Mohammed AlJumah; jumahm@gmail.com

Received 22 June 2021; Accepted 22 October 2021; Published 9 November 2021

Academic Editor: Francesco Patti

Copyright (c) 2021 Reem Bunyan et al. This is an open access article distributed under the Creative Commons Attribution License, which permits unrestricted use, distribution, and reproduction in any medium, provided the original work is properly cited.

Background. The prevalence of multiple sclerosis (MS) appears to be increasing worldwide. However, data on the pediatric onset of MS is lacking, particularly in developing countries. Objective. This study is aimed at reporting the current burden of the pediatric onset of MS in the five regions of Saudi Arabia. Methods. This study used relevant data from the National Saudi MS Registry that was operational between 2015 and 2018. The data on patients with pediatric onset MS from all the hospitals included in the registry was retrospectively analyzed using the age of diagnosis. Patients who were 1-18 years old when diagnosed were included in the analysis. Results. The registry included 287 patients with pediatric onset MS, with a mean age of diagnosis at 15.7 (SD: 2.06). 74.2\% of the participants were females. For the included hospitals, the estimated prevalence of pediatric MS was at 2.73/100,000 pediatric Saudi population. The prevalence of pediatric MS in the remaining nonparticipant hospitals was then projected taking into account both the size of pediatric population in the Kingdom per region and the number of facilities treating and managing MS in each of the corresponding regions. The overall projected prevalence was found to be 14.33/100,000 Saudi pediatric population. Conclusion. To the best of our knowledge, this study reported the latest 
epidemiological data of pediatric onset of MS in Saudi Arabia. The current prevalence of MS among the pediatric Saudi population was found to be $2.73 / 100,000$, and the overall projected prevalence was estimated at $14.33 / 100,000$. Our findings were similar to those in other pediatric MS cohorts. Further studies are needed to understand the long-term prognosis, response to treatment, and disease course.

\section{Introduction}

Multiple sclerosis (MS) is recognized as a disease affecting predominantly adults. However, in the 1980s, pediatric onset of MS became recognized, and more attention has been directed towards the disease in this age group, its diagnosis, and management [1]. A recent review of published data on pediatric onset of MS during 1965-2018 reported that incidence of pediatric onset of MS is $0.05-2.85 / 100,000$, and the prevalence is $0.69-26.92 / 100,000$ [2].

The age of onset of MS typically ranges between 20 to 40 years; however, pediatric onset of MS is known to occur before the age of 18 years [2-5]. Pediatric onset multiple sclerosis occurs during the "key formative years of education and during the period of active brain maturation" [6]. Findings from number of studies suggest that it may have lasting consequences on affected individuals and potentially disrupting their ability to effectively study, work, and engage in society, emphasizing the need for understanding and discovery of specific clinical or demographic risk factors and eventually protective and supportive strategies for all patients with multiple sclerosis [6,7].

Various factors are associated with the pediatric onset of MS. Exposure to viruses, such as Epstein-Barr virus (EBV), has been reported in children that later developed MS. Other factors include childhood obesity, vitamin D deficiency, and metabolic syndrome [8, 9]. Genetic factors have also been implicated in pediatric onset MS as in adult onset MS [10, 11].

The predominant clinical features of pediatric onset MS are headache, nausea, vomiting, fever, seizures, an altered level of consciousness, motor, sensory, and cerebellar and brainstem dysfunction [1]. Studies have reported good recovery among children compared to adults, with the majority recovering after their first attack and very few children suffering from disability $[12,13]$.

In Saudi Arabia, adult onset MS is more systematically studied; however, there is a shortage in the epidemiology of pediatric onset of MS. This article is aimed at reporting the prevalence of pediatric onset MS using data from the National Saudi MS Registry, in addition to describe relevant disease characteristics in that population, such as symptoms at onset, disease course, and family history which in turn may provide valuable information to better understand the actual burden of the disease, the risks for development, and progression. Using national hospital-based registries and databases to report burden of disease is known to help future planning and policymaking at both levels, regional and national [14]. Despite the low prevalence of pediatric onset MS globally, it is important to understand its epidemiology in the Kingdom as it could help in decision-making and various clinical practices.

\section{Methods}

The study utilized data collected earlier by the National MS Registry that was launched in 2015 and lasted until 2018. Detailed description of the registry can be found elsewhere $[14,15]$. Physicians in participating hospitals entered patients' disease related data into a web-based application. Data on people diagnosed with MS at the age of 18 year or younger ( $\leq 18$ years) were included in this study.

The frequency distribution tables were generated according to the five major regions in the Kingdom (Western, Central, Eastern, Southern, and Northern Regions). Demographics, clinical characteristics, affected CNS regions, and family history were summarized using descriptive statistics, such as mean (SD), median (interquartile range), or frequency (percentage).

Projections for the overall prevalence were performed at regional and national levels, following the same methodology used in our previously published paper that described the overall prevalence of the disease in KSA and utilizing the same registry database [14]. The overall projected prevalence of pediatric onset MS in the Kingdom was calculated and extrapolated taking into account the number of registered patients with pediatric onset MS in the included hospitals in the registry (20 hospitals) in each region and assuming a similar number of cases in the remaining nonparticipant hospitals for the same region, in addition to the total number of pediatric population in each region [14].

The total pediatric population in Saudi Arabia (the denominator for projected overall prevalence) was last recorded at 10533635 in 2018 [16]. Finally, it is noteworthy to point out that the regional actual and projected prevalence were calculated based on the patients' region of residence rather than the hospitals region where they were registered.

\section{Results}

The Saudi National Registry described in a previous study [14] included 287 patients with pediatric onset MS from the different regions of the Kingdom. The median age of MS diagnosis was 16.0 years (IQR: $15.0-17.0$ ), and $74.2 \%$ of patients were females. For the included hospitals in the registry, the prevalence of pediatric MS was estimated at 2.73/100,000 pediatric Saudi population. Based on these estimates, the prevalence of pediatric MS in the remaining nonparticipant hospitals was then projected considering both the size of pediatric population in the Kingdom per region and the number of facilities treating and managing MS patients in each of the corresponding regions as shown in Table 1.

Finally, the overall prevalence was estimated and found to be $14.33 / 100,000$ Saudi pediatric population as shown in 
TABle 1: Total Saudi pediatric population, number of facilities treating MS patients, and number of facilities included in the National MS Registry, Saudi Arabia.

\begin{tabular}{lccc}
\hline Regions & $\begin{array}{c}\text { Pediatric } \\
\text { population }\end{array}$ & $\begin{array}{c}\text { Hospitals/facilities } \\
\text { treating MS } \\
\text { patients in KSA }\end{array}$ & $\begin{array}{c}\text { Hospitals/ } \\
\text { facilities } \\
\text { included in } \\
\text { registry }\end{array}$ \\
\hline Western region & 3387191 & 30 & 7 \\
Central region & 3047059 & 36 & 7 \\
Eastern region & 1516795 & 22 & 4 \\
Southern region & 1694334 & 9 & 2 \\
Northern region & 888256 & 8 & 0 \\
Total “2018” & 10533635 & 105 & 20 \\
\hline
\end{tabular}

*Table adapted from AlJumah, M., Bunyan, and R., Al Otaibi, H. et al. (2020).

Table 2. The prevalence of pediatric onset MS varied between the different regions in the Kingdom and was found to be the highest for the western region. Projected prevalence with respect to regions was found to be different, with the highest prevalence reported in the eastern region followed by the western, northern, central, and southern.

Out of the total number of patients with pediatric onset MS, $39.4 \%$ were from the western region, $34.1 \%$ from the central followed by $17.4 \%$ from the east, $5.2 \%$ from the south, and $3.8 \%$ from the northern region as shown below in Table 3.

Table 4 shows that the most common symptoms at onset as reported by participants were motor weakness $(55.1 \%)$ followed by visual and sensory symptoms $(50.5 \%$ and $41.8 \%$, respectively). Among all patients, the majority had a polysymptomatic presentation with $69.3 \%$ presenting with two symptoms, $10.3 \%$ with three, $2.4 \%$ with four, and $1.2 \%$ with five. The CNS region affected was documented among $85.3 \%$ of patients at the time of enrolment in the registry, and the most commonly affected region was the cerebellar $(31.0 \%)$ followed by the visual and spinal pathway $(28.9 \%$ and $20.9 \%$, respectively). Concomitant diseases were reported among $9.8 \%$ of patients.

Brain MRI scans were carried out for $85.4 \%$ of the patients, spine MRI in 79\% (cervical MRI for $35.5 \%$, whole spine MRI for $29.3 \%$, and thoracic MRI for $14.3 \%$ ) as seen in Figure 1 below. Evoked potentials were carried out in $17.8 \%$ of patients and lumbar puncture in $23.3 \%$. Of the total, $38.0 \%$ patients had no history of relapse, $87.7 \%$ were using medication for relapse, and $80.0 \%$ were taking disease-modifying therapy (DMTs).

Among those on DMTs, majority used Rebif (interferon beta-1a) (28.0\%), followed by Betaserone (interferon beta1b) (21.6\%), Avonex (interferon beta-1a) (16.8\%), Tysabri (natalizumab) (13.8\%), and Gilenya (fingolimod) (13.4\%) as shown in Figure 2.

Of the total patients enrolled in the registry, $94.0 \%$ were diagnosed with relapsing remitting MS. The mean EDSS score reported at the time of inclusion was 1.67 (SD: 2.12). Table 5 shows that family history of MS was present among $17.5 \%$ of patients, with parental consanguinity among
$33.9 \%$, and $7.4 \%$ reporting that their siblings were also affected with MS.

\section{Discussion}

To the best of our knowledge, this is the first study to provide the current estimates of prevalence of pediatric onset of MS in Saudi Arabia. An earlier study by AlJumah, Bunyan, Al Otaibi et al. (2020), which was derived from the same registry, reported the overall prevalence of MS in the Kingdom and projected the overall prevalence to be $40.40 / 100,000$ population, while the current study presents preliminary findings specific to the burden of pediatric onset of MS in the Kingdom and projected the pediatric onset overall prevalence to be $14.33 / 100,000$ Saudi pediatric population. Although it has been established that the prevalence of multiple sclerosis (MS) seems to be increasing worldwide, data on the pediatric onset of MS is still lacking in general.

MS is a disease that mainly affects adults, but it can also affect children. Globally, the incidence and prevalence of pediatric onset of MS are low; however, an increase in the rates of both the incidence and prevalence of the pediatric onset of MS has been reported [5]. Thus, this article is aimed at reporting the current epidemiology in Saudi Arabia as a whole and at a regional level of the country.

The current prevalence reported in our study estimated at 2.73 was much lower than that reported from the Kuwaiti registry, which was estimated at 6.0/100,000 [17], however, closer to prevalence reported in other studies [2]. The median age of onset of our cohort was 15.0 years, which was quite similar to what was seen in Kuwait (15.4 years), but higher than what was reported in the western world. For example, the UK reported that the mean age of onset was 9.7 years, while Canada reported 10.5 years and France 9.9 years [17-20]. Female predominance was reported globally and regionally, which was supported by the findings in this study, in which two thirds of the patients were females [17-20].

Variation in the findings could be related to the high rates of MS internationally, evolution of the diagnostic criteria, the use of surveillance data, and other methodological variations. The findings of this study were extracted from the data of a well-managed registry that included data from all regions of Saudi Arabia. Likewise, the suggested upward trend in the prevalence of the pediatric onset of MS in Saudi Arabia could have several explanations. Firstly, the overall increasing trend in the prevalence of pediatric onset MS globally, secondly, the increased awareness and improved diagnostic tools that may have helped in the early detection and identification of these patients, and lastly, the establishment of the national registry that has helped in the proper identification and reporting of cases, thus maintaining a record for reporting true estimations.

However, the reported prevalence in our study should be concluded cautiously for multiple reasons. For instance, the registry included data from $19 \%$ of hospitals treating the MS population in the Kingdom. In addition, the projected prevalence was estimated under the assumption that the 
TABLE 2: Total Saudi pediatric population and prevalence of pediatric MS among Saudi pediatric population.

\begin{tabular}{lcccc}
\hline Regions & Total Saudi pediatric population & $\begin{array}{c}\text { Patients with pediatric onset } \\
\text { MS (from participant hospitals*) }\end{array}$ & $\begin{array}{c}\text { Prevalence/100,000 } \\
\text { pediatric population }\end{array}$ & $\begin{array}{c}\text { Projected prevalence/ } \\
100,000 \text { population }\end{array}$ \\
\hline East & 1516795 & 50 & 3.30 & 18.13 \\
West & 3387191 & 113 & 3.31 & 14.71 \\
North** & 888256 & 11 & 1.24 & 9.91 \\
Central region & 3047059 & 98 & 3.22 & 16.56 \\
South & 1694334 & 15 & 0.89 & 3.98 \\
Total "2018" & 10533635 & 287 & 2.73 & 14.33 \\
\hline
\end{tabular}

${ }^{*} 20$ hospitals participated in the registry. ${ }^{* *}$ Given that there were no participating hospitals from the northern region in the registry, the assumption was made that the 11 patients enrolled in the registry from the northern region were referred to hospitals in other regions.

TABLE 3: Baseline characteristics of individuals with pediatric onset of MS $(n=287)$.

\begin{tabular}{lcc}
\hline Demographics & Frequency & Percentage \\
\hline Age & & \\
$\quad$ Median IQR & $16.0(15.0-17.0)$ & \\
<12 years & 14 & 4.9 \\
$\quad$ 12-18 years & 273 & 95.1 \\
Gender & & \\
Male & 74 & 25.8 \\
Female & 213 & 74.2 \\
Regions & & \\
West & 113 & 39.4 \\
Central & 98 & 34.1 \\
East & 50 & 17.4 \\
North & 11 & 3.8 \\
South & 15 & 5.2 \\
\hline
\end{tabular}

prevalence will be the same across the remaining hospitals, which could impact the findings by slightly under or overestimating the true picture. Finally, the pediatric onset of MS was reported retrospectively by using the date of diagnosis, which in turn could affect the actual burden of the disease. Despite all these limitations, the study showed the current estimates and provided important insights that may help policy/decision makers to better address the potentially growing rates of pediatric MS in the Kingdom.

Overall, the rates of the pediatric onset of MS in Saudi Arabia are not significantly higher when compared with western countries where the prevalence of MS is generally high. However, an increasing trend could be seen throughout the country in the future. Gender disparity and other signs and symptoms should be kept in consideration while diagnosing and treating pediatric onset of MS. Through this study, we were able to provide initial epidemiological data and insights on pediatric onset MS in Saudi Arabia and the prevalence at a regional level and country level. These findings could be the baseline foundation for future studies and an array of future research initiatives. Further longitudinal studies targeting the long-term outcomes, relapse, and management are warranted to build upon the findings of this study.
TABLE 4: Signs and symptoms and the involvement of CNS region among individuals with pediatric onset MS $(n=287)$.

\begin{tabular}{lcc}
\hline Symptoms and affected regions & Frequency & Percentage \\
\hline Symptoms of $1^{\text {st }}$ attack & 158 & \\
Motor weakness & 5 & 55.1 \\
Bulbar symptom & 18 & 1.7 \\
Bladder and bowel difficulty & 11 & 6.3 \\
Neuropsychological function & 79 & 3.8 \\
Ataxia & 120 & 27.5 \\
Sensory symptoms & 145 & 41.8 \\
Visual symptoms & 38 & 50.5 \\
Fatigue & 25 & 13.2 \\
Pain & 8 & 8.7 \\
Seizures & & 2.8 \\
CNS region affected & 243 & \\
Any region affected & 83 & 85.3 \\
Visual pathway & 60 & 28.9 \\
Spinal cord & 43 & 20.9 \\
Brainstem & 89 & 15.0 \\
Cerebellar & 30 & 31.0 \\
Pyramidal tract & 17 & 10.5 \\
Bowel & 44 & 5.9 \\
Sensory function & 64 & 15.3 \\
Multiple regions & & 22.3 \\
\hline
\end{tabular}

Our study showed the median age at presentation to be 15.0 years similar to findings in other pediatric MS cohorts in the postpubertal age, with only $4.9 \%$ of patients younger than the age of 12 years. The Saudi Health Council defines the pediatric age group to be from birth up to 14 years and adolescence from 14 to 18 years. There are also variations in the age at which patients' transition from pediatric to adult care providers. These factors might influence patterns in diagnosis and care for pediatric onset MS in Saudi Arabia. Further studies are needed to assess this variation within the Kingdom's regions and among respective hospitals.

The clinical phenotype found in our study was similar to that in other pediatric MS cohorts with a common polysymptomatic presentation. The most common initial symptom of the disease was motor weakness in $55.1 \%$ of patients, 


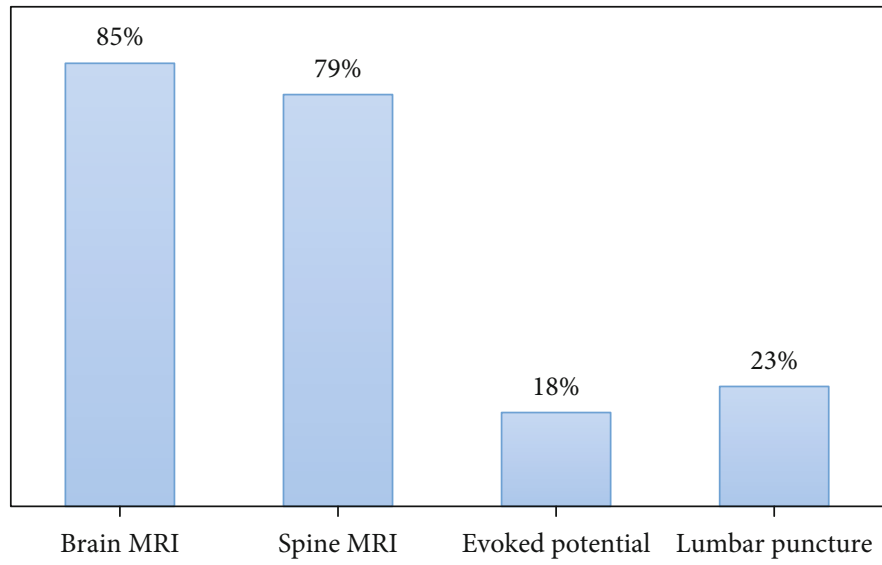

Figure 1: Diagnostic tests among individuals with pediatric onset of MS ( $n=287)$.

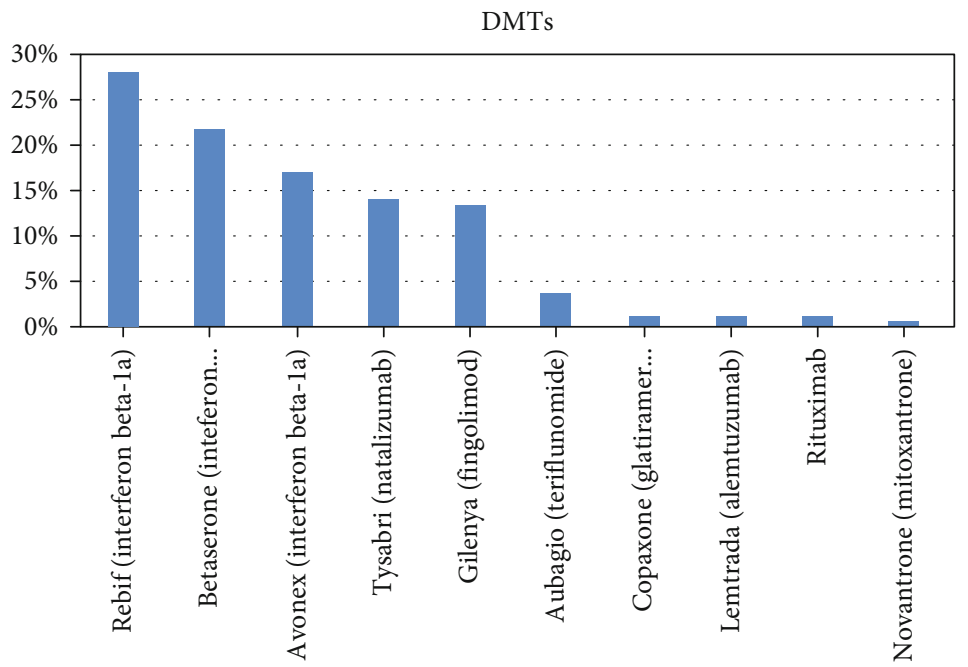

FIGURE 2: DMTs among patients with pediatric onset of MS $(n=232)$.

TABLE 5: Family history of MS among individuals with pediatric onset of MS $(n=287)$.

\begin{tabular}{lcc}
\hline Family history & Frequency & Percentage \\
\hline Family history of MS* & 50 & \\
$\quad$ Yes & 235 & 17.5 \\
No & & 82.5 \\
Parental consanguinity* & 92 & \\
Yes & 179 & 33.9 \\
No & & 66.1 \\
Sibling affected & \\
Yes & 19 & 7.4 \\
No & 239 & 92.6 \\
\hline
\end{tabular}

* indicates missing data.

followed by visual symptoms in $50.5 \%$, and sensory symptoms in $41.8 \%$. The most common CNS region affected initially was cerebellar, followed by visual in $28.9 \%$. These findings are also similar to those in the literature from other pediatric cohorts. None of the patients reported having symptoms related to altered level of consciousness, seizures, or a prior diagnosis of acute disseminated encephalomyelitis (ADEM). The mean EDSS score at the time of enrollment in the registry was low. Longitudinal studies are needed to study disability among these patients and how they compare with adult onset disease.

\section{Conclusion}

This study reported the epidemiology of pediatric onset of MS in Saudi Arabia utilizing a national disease registry. The current prevalence of MS among the pediatric Saudi population was found to be $2.73 / 100,000$, and the overall projected prevalence was estimated at $14.33 / 100,000$. Our findings were similar to those in other pediatric MS cohorts. Further studies are needed to understand the long-term prognosis, response to treatment, and disease course.

5.1. Study Limitation. Data on MS patients was not collected from all hospitals diagnosing and treating MS population in the Kingdom; however, the included hospitals in the registry 
were comparable in their characteristics to those not included (such size, catchment area, number of beds, average number of neurologists). Therefore, the prevalence of pediatric MS was a projection based on the assumption that number of diagnosed MS cases in included/participating hospitals in the registry (in each region) is similar to those in nonparticipating hospitals in the same region. While this limitation may impact the generalizability of the findings, nevertheless, the study represents a good initial effort in establishing current estimates of prevalence of pediatric onset of MS in Saudi Arabia. Further and more comprehensive studies are warranted to get more generalizable results.

\section{Abbreviations}

$\begin{array}{ll}\text { MS: } & \text { Multiple sclerosis } \\ \text { KSA: } & \text { Kingdom of Saudi Arabia } \\ \text { EDSS: } & \text { The Expanded Disability Status Scale } \\ \text { CNS: } & \text { Central nervous system } \\ \text { ADEM: } & \text { Acute disseminated encephalomyelitis }\end{array}$

\section{Data Availability}

Due to the nature of the data involved in this study, access to this data is restricted and requires appropriate approvals from the authorized and participating parties.

\section{Conflicts of Interest}

The authors declare that there is no conflict of interest regarding the publication of this paper.

\section{Acknowledgments}

This study and the registry on which it was based were supported by King Abdulaziz City for Science and Technology.

\section{References}

[1] N. Gadoth, "Multiple sclerosis in children," Brain \& Development, vol. 25, no. 4, pp. 229-232, 2003.

[2] A. Jeong, D. M. Oleske, and J. Holman, "Epidemiology of pediatric-onset multiple sclerosis: a systematic review of the literature," Journal of Child Neurology, vol. 34, no. 12, pp. 705-712, 2019.

[3] B. Banwell, A. Ghezzi, A. Bar-Or, Y. Mikaeloff, and M. Tardieu, "Multiple sclerosis in children: clinical diagnosis, therapeutic strategies, and future directions," Lancet Neurology, vol. 6, no. 10, pp. 887-902, 2007.

[4] M. Ruggieri, P. Iannetti, A. Polizzi, L. Pavone, and L. M. E. Grimaldi, "Multiple sclerosis in children under 10 years of age," Neurological Sciences, vol. 25, no. S4, pp. s326-s335, 2004.

[5] R. Alroughani and A. Boyko, "Pediatric multiple sclerosis: a review," BMC Neurology, vol. 18, no. 1, p. 27, 2018.

[6] A. Waldman, A. Ghezzi, A. Bar-Or, Y. Mikaeloff, M. Tardieu, and B. Banwell, "Multiple sclerosis in children: an update on clinical diagnosis, therapeutic strategies, and research," Lancet Neurology, vol. 13, no. 9, pp. 936-948, 2014.

[7] K. A. McKay, E. Friberg, N. Razaz, K. Alexanderson, and J. Hillert, "Long-term Socioeconomic Outcomes Associated
With Pediatric-Onset Multiple Sclerosis," JAMA Neurology, vol. 78, no. 4, p. 478, 2021.

[8] M. A. Gianfrancesco, P. Stridh, B. Rhead et al., "Evidence for a causal relationship between low vitamin $\mathrm{D}$, high $\mathrm{BMI}$, and pediatric-onset MS," Neurology, vol. 88, no. 17, pp. 1623$1629,2017$.

[9] P. Brambilla, I. Lissau, C.-E. Flodmark et al., "Metabolic riskfactor clustering estimation in children: to draw a line across pediatric metabolic syndrome," International Journal of Obesity, vol. 31, no. 4, pp. 591-600, 2007.

[10] C. Lucchinetti, W. Brück, J. Parisi, B. Scheithauer, M. Rodriguez, and H. Lassmann, "Heterogeneity of multiple sclerosis lesions: implications for the pathogenesis of demyelination," Annals of Neurology, vol. 47, no. 6, pp. 707-717, 2000.

[11] M. Tardieu and Y. Mikaeloff, "Multiple sclerosis in children," International MS Journal, vol. 11, no. 2, pp. 36-42, 2004.

[12] C. L. de Mol, Y. Wong, E. D. van Pelt et al., "Incidence and outcome of acquired demyelinating syndromes in Dutch children: update of a nationwide and prospective study," Journal of Neurology, vol. 265, no. 6, pp. 1310-1319, 2018.

[13] N. Krajnc, J. Oražem, Z. Rener-Primec, and M. Kržan, "Multiple sclerosis in pediatric patients in Slovenia," Multiple Sclerosis and Related Disorders, vol. 20, pp. 194-198, 2018.

[14] M. AlJumah, R. Bunyan, H. al Otaibi et al., "Rising prevalence of multiple sclerosis in Saudi Arabia, a descriptive study," BMC Neurology, vol. 20, no. 1, p. 49, 2020.

[15] M. AlJumah, H. Al Otaibi, G. al Towaijri et al., "Familial aggregation of multiple sclerosis: results from the National Registry of the Disease in Saudi Arabia," Multiple Sclerosis JournalExperimental, Translational and Clinical, vol. 6, no. 4, article 205521732096049, 2020.

[16] “General Authority for Statistics. Annual Report 2018," http:// www.stats.gov.sa/en.

[17] R. Alroughani, S. Akhtar, S. Ahmed, R. Behbehani, J. al-Abkal, and J. al-Hashel, "Incidence and prevalence of pediatric onset multiple sclerosis in Kuwait: 1994-2013," Journal of the Neurological Sciences, vol. 353, no. 1-2, pp. 107-110, 2015.

[18] S. D. Brass, Z. Caramanos, C. Santos, M.-E. Dilenge, Y. Lapierre, and B. Rosenblatt, "Multiple sclerosis vs acute disseminated encephalomyelitis in childhood," Pediatric Neurology, vol. 29, no. 3, pp. 227-231, 2003.

[19] B. Banwell, J. Kennedy, D. Sadovnick et al., "Incidence of acquired demyelination of the CNS in Canadian children," Neurology, vol. 72, no. 3, pp. 232-239, 2009.

[20] Y. Mikaeloff, G. Caridade, S. Assi, S. Suissa, M. Tardieu, and on behalf of the KIDSEP Study Group, "Prognostic factors for early severity in a childhood multiple sclerosis cohort," Pediatrics, vol. 118, no. 3, pp. 1133-1139, 2006. 